\title{
Genetic markers in HLA region to identify patients with tuberculosis risk, in RA subjects
}

Objetive: To identify the patients with RA at highest risk of TB, prior to the use of biological therapy antiTNF.

Method: We carried out a study of two phases considered three groups, in the first we performed a case and control study in the Hospital Juárez de México, in the second phase, the results of phase one were analyzed with those obtained in the group of patients identified with Poncet's disease. We compared the classes I and II of the HLA of patients naïve to biologic treatment with healthy controls.

Result: We have identified several candidate alleles that will help to advance the personalized treatment of RA that takes into account the genetic risk to develop TB, particularly the alleles B27 and DQB1*0301.

Conclusion: Patients with RA have an increased risk to develop TB and severe complications derived from it. This infectious process is related to the use of biologic.

Keywords: tuberculosis $\bullet$ biologics $\bullet$ rheumatoid arthritis $\bullet$ HLA

\section{Introduction}

Biologics like the inhibitors of tumor necrosis factor $\alpha$ (TNF- $\alpha)$, antibodies directed towards several interleukins, $\mathrm{T}$ cells, B cells and JAK inhibitors have positively impacted the lives of patients with inflammatory and autoimmune diseases [1]. For instance, patients with rheumatoid arthritis show an improvement in clinical and functional outcomes, including quality of life. However, the use of biologics has been consistently associated to an increased risk of opportunistic diseases [2].

Tuberculosis (TB) is the most important infectious disease that erupts in patients that use biologics [3]. This mycobacterial infection causes more than 2.5 million of deaths in a year. Moreover, approximately one third of the world's population is infected with Mycobacterium tuberculosis (M. tb) making it a public health concern [4]. Despite the very high number of infected patients, less than 10\% of them develop the active form of the disease because of the interaction between the bacteria, the environment and the host [5]. Among the risk factors of the host that determine the susceptibility of the disease are those that cause an acquired or a primary immunodeficiency. Besides malnutrition, alcoholism and poverty, the use of biologics are a major cause of secondary immunodeficiency in patients with RA [6]. A disproportionate amount of patients that receive infliximab, a TNF- $\alpha$ inhibitor, contact tuberculosis [7]. Thanks to some preventive strategies, this risk has fallen in recent years.

Numerous attempts to dampen the risk of infection or re-activation of tuberculosis (TB) in patients that take biologics have been conducted. For instance, the Mantoux reaction is used in countries where the prevalence of $\mathrm{TB}$ is low, whereas other screening methods that use the interferon gamma produced by the macrophages are employed in regions of the World where TB is endemic, like Mexico [3]. Likewise, prophylactic therapy for TB should be completed before the initiation of a biologic therapy [3]. However, there is still a population of patients that develop the active form of the disease despite screening and profilaxis [8]. Therefore, there is a need to understand which patients are at risk of developing TB in order to select appropriate therapies and avoid the suffering and cost that this disease causes.

Genomic markers are among the best candidates to inform which patients are at increased risk of developing TB [9]. For instance, around a great amount of the risk of TB has been attributed to genetic factors [10]. The latter is explained because the immune response to $M$. tb is determined by the genetic composition of the host [11]. Of the genes that are relevant to fight intracellular infections, the Human Leukocyte Antigen (HLA) is of particular importance.

\author{
Gustavo Esteban Lugo- \\ Zamudio*1, Rosa Elda Barbosa- \\ Cobos', Dolores Delgado- \\ Ochoa', Rodrigo Huerta- \\ Gutiérrez $^{2} \&$ Julio Granados \\ Arreola $^{2}$ \\ 'Hospital Juárez de México, México City, \\ México \\ ${ }^{2}$ Division of Immunogenetics, Transplan \\ Departament, Instituto Nacional de \\ Ciencias y Nutrición Salvador Zubirán, \\ México City, México \\ *Author for correspondence: \\ gelz1@prodigy.net.mx
}


The function of the class I and class II alleles goes beyond antigen presentation to the T-cells and has in fact several immunomodulatory properties. The latter and its hyper-polymorphic nature make the HLA an excellent candidate to study population genetics. For instance, the presence of HLA-DR2 has been associated to pulmonary $\mathrm{TB}$ in several populations [1214]. Moreover, other alleles like $\mathrm{DRB}^{*} 16$, DRB1*1501 and DQB1*0503 have also been involved [15]. Besides representing a risk factor for TB, the HLA also mediates the interaction between RA and the infection.

The association of TB with Rheumatoid Arthritis (RA) was recognized decades ago. Although the involvement of M. tb in the etiology of RA has been abandoned, other forms of interaction have been proposed. For instance, in the particular case of Poncet's disease (tuberculous rheumatism), the alleles B27 and DQB1*0301 play an important role, which supports the idea of a genetic predisposition to develop RA in patients that are exposed to mycobacterial antigens [16]. Moreover, autoimmune diseases are recognized as ruptures on the mechanisms of tolerance which may impact the infection status. Genetic data has supported the existence of several RA phenotypes [17]; each of them may respond differently to infectious insults. Finally, genetics can also impact the response of biologic therapy, which in turn may affect the development of TB.

To identify patients with RA that have a higher risk of developing $\mathrm{TB}$, we determined their class I and II alleles of the HLA and compared them with those of a group of healthy controls matched for age and sex. We hypothesized that the patients with protective alleles for TB would be better candidates to receive biologic therapy.

\section{Materials and methods}

\section{Population}

We carried out a study of two phases considered three groups; in the first we performed a case and control study in the Hospital Juárez de México. We included 120 patients of the Department of rheumatology with the diagnosis of RA according to the American College of Rheumatology (ACR) criteria established in 1987. The patients were naïve to biological treatment at the beginning of the study and were candidates to receive it. As controls, we used 120 healthy subjects taken from the database of the program of related living kidney donor. The population belongs to Mexican mestizo ethnicity, according to the trihybrid model (admixture of Amerindian, European and African populations) [18]. The subjects were born in Mexico and have a family history of Mexican ascendance in at last three generations, in the second phase, the results of phase one were analyzed with those obtained in the group of patients identified with Poncet's disease, diagnosed by Polymerase Chain Reaction (PCR) specific sequence primer using Taq polymerase (Promega, Madison, WI, USA).

The research protocol was approved by internal review board of the HJM with a registration number 0065/15R. All the participants signed an informed consent that includes the management of genetic material.

\section{Laboratory procedures}

Research subjects were genotypified for HLA class I (A and B) and class II (DRB1 and DQB1) using PCR-SSP (Invitrogen ABDRDQ SSP UniTray MR, Life Technologies Corporation Brown Deer, Wisconsin, EE.UU. MR). The alleles were validated with using the IMGT/HLA database [19] that allows retrieving the information about one particular allele as mentioned in WHO's Committee of Nomenclature [20].

\section{Statistical analysis}

Allele and haplotype frequencies and HardyWeiberg equilibrium were estimated using the Arlequin software v.3.1. and MedCalc SoftwareMR 1993-2015 version 15.8, modified on August $14^{\text {th }}$ of 2015. The strength of the association was given as an Odds Ratio (OR) with a 95\% Confidence Interval (CI), and $\mathrm{p}$ values of 0.05 were considered significant.

\section{Results}

The study included 120 patients diagnosed with RA naïve to biologic therapy at risk of developing TB, 78 subjects $(65 \%)$ where women and 42 (35\%) men, and 120 healthy controls from the study group, 70 subjects were women $(58 \%)$ and 50 individuals $(42 \%)$ were men. The age of the population ranged from 18 to 60 years old.

\section{Frequency of alleles that confer risk to TB}

In the study population, we found $26(21.6 \%)$ patients and $13(10.8 \%)$ controls with the allele $\mathrm{DQB} 1{ }^{*} 0501$. Moreover, the frequency of the allele DRB1*1501 was of $6(5 \%)$ and $10(10 \%)$ in patients as compared to controls. Other allele associated with an increased risk for TB is $\mathrm{DQB1}{ }^{*} 0301$; its frequency in patients was of $55.8 \%$ whereas in controls it was of $39.2 \%$. On 
the other side, DQB1*0601 showed a frequency of $16.6 \%$ and $39.2 \%$ in patients and controls, respectively.

\section{Frequency of alleles that confer protection to TB}

The proportion of patients positive for one of the variants of the DR4 serotype was equal in patients and in controls (43.3\%). For the case of the serotype DR8, $8 \%$ of the patients and $18.3 \%$ of the controls had the DRB $1^{*} 0801$ allele. On the other side, $6 \%$ of patients and $21 \%$ of controls had a DRB*11 allele.

\section{HLA polymorphisms and risk of RA}

Significant differences were found the frequencies of several alleles between patients and controls. Table 1 reports the odds ratio, the confidence interval and the $\mathrm{p}$-value for each allele. The alleles that patients exhibited in a frequency more elevated than expected were: $A^{*} 24: 02$, $\mathrm{A}^{*} 33: 01, \mathrm{~B}^{*} 0801, \mathrm{DQB} 1{ }^{*} 0301, \mathrm{DQB} 1^{*} 0403$, DQB1*0501, DRB1*0302 and DRB1*1402.

HLA polymorphisms and protection from RA

Disproportionate frequencies were associated to a lower prevalence of RA as compared to controls. Table 2 reports the odds ratio, confidence intervals and p-values for each allele. The alleles that were more prevalent in controls were: $A^{*} 02: 50, A^{*} 29: 01, A^{*} 30: 01, A^{*} 29: 01, A^{*} 30: 01$, DQB1*0601, DRB1*0801 and DRB1*1404.

\section{Discussion}

This study genotyped the HLA locus of 120 patients with RA that were naive to biological treatment and had an increased risk of developing TB and 120 healthy controls. Among the entire population, the prevalence of at least one allele that conferred risk for developing TB was of $98 \%$. On the other side, $71 \%$ of the population had at least one protective allele against TB [2], the allele HLA-DQB1*0501 was present in $21.6 \%$ of the patients with RA. This allele was previously reported to increase the risk of presenting TB in Mexican patients [21]. However, a recent metaanalysis including patients of diverse populations concluded that DQB1*0501 was not significantly associated with risk of this disease [22]. The different results could be due to the small proportion of Amerindian individuals that were included in the meta-analysis. Interestingly, DQB1*0501 also contributed to the risk of RA which is in accordance to previous findings. Furthermore, this allele is associated with pulmonary complications of RA. Although TB in patients that use biologics is predominantly extra-pulmonary, the complications of this organ related to $\mathrm{DQB1}{ }^{*} 0501$ may represent a physiopathogenic pathway for the disproportionately increased risk of TB.

Another class II allele associated with an increased risk of $\mathrm{TB}$ is $\mathrm{DQB} 1{ }^{*} 0301$ which frequency in patients was of $55.8 \%$. Interestingly, the presence of this allele also posed risk for RA in our population. In fact the alleles B27 and DQB1*0301 were identified as part of the genetic elements that favor the development of Poncet's disease [16]. This evidence is aligned with the hypothesis of a causal relation of this allele with the development of autoimmunity. If the infection with $M . t b$ was confirmed in an elevated proportion of carriers of DQB $1^{*} 0301$, the diagnosis of RA may be challenged, especially if symptoms change after antifimic treatment (Table 3).

Although the frequency of the allele DRB1*1501 was low (5\%), it has been associated with TB in Mexican (Granados) and in Indian populations [23]. Furthermore, the allele DRB1*15 was associated with $\mathrm{TB}$ in a recent meta-analysis, which confirms the strength and consistency

\begin{tabular}{|c|c|c|c|c|}
\hline HLA ALLELE & PACIENTS $n=120$ & CONTROLS $n=120$ & OR (IC) ${ }^{\circ}$ & $p<0.05$ \\
\hline$A * 02: 50$ & 8 & 24 & $0.28(0.12$ a 0.66 & 0.0037 \\
\hline$A * 29: 01$ & 1 & 12 & 0.075 ( 0.0097 a 0.59$)$ & 0.014 \\
\hline$A * 30: 01$ & 10 & 22 & 0.4 ( 0.18 a .089$)$ & 0.025 \\
\hline$B^{*} 0801$ & 10 & 1 & $10.8(1.36$ a 85.8$)$ & 0.024 \\
\hline DQB1*03:01 & 67 & 47 & 1.9 (1.17 a 3.28$)$ & 0.01 \\
\hline DQB1*04:03 & 9 & 0 & 20.5 (1.18 a 356.9) & 0.038 \\
\hline DQB1*05:01 & 26 & 13 & 2.27 (1.10 a 4.6) & 0.0254 \\
\hline DQB1*06:01 & 20 & 47 & $0.31(0.16$ a 0.56$)$ & 0.0001 \\
\hline DRB1 ${ }^{*} 14: 02$ & 15 & 3 & 5.5 ( 1.5 a 19.7) & 0.007 \\
\hline DRB1*14:04 & 0 & 7 & $0.06(.003$ a 1.11$)$ & 0.05 \\
\hline DRB $1 * 14: 10^{*}$ & 10 & 3 & $3.5(0.9$ a 13.22$)$ & 0.05 \\
\hline
\end{tabular}




\begin{tabular}{|c|c|c|c|c|}
\hline \begin{tabular}{|l|} 
HLA CLASE \\
ALLELES I Y II
\end{tabular} & $\begin{array}{l}\text { PACIENTS } \\
n=18\end{array}$ & CONTROLS $n=120$ & OR (IC) ${ }^{\circ}$ & $\mathbf{p}<0.05$ \\
\hline$A * 24: 02$ & 6 & 23 & 1.85 (0.97 a 3.5$)$ & 0.05 \\
\hline$B * 0801$ & 2 & 1 & 10.8 (1.36 a 85.8$)$ & 0.024 \\
\hline$B * 15: 55$ & 1 & 0 & $44.5(2.39-829)$ & 0.01 \\
\hline$B * 27: 01$ & 1 & 0 & $44.5(2.39-829)$ & 0.01 \\
\hline$B * 35: 01$ & 3 & 9 & $2.6(1.3-5.3)$ & 0.009 \\
\hline$B * 35: 02$ & 1 & 0 & $44.5(2.39-829)$ & 0.01 \\
\hline$B * 37: 01$ & 1 & 0 & $44.5(2.39-829)$ & 0.01 \\
\hline$B * 38: 03$ & 3 & 0 & $70(4.0-1216)$ & 0.0035 \\
\hline DQB1*0301 & 18 & 0 & $8917(171-4633)$ & 0.0009 \\
\hline DQB1*02:01 & 1 & 7 & $44.5(2.39-829)$ & 0.01 \\
\hline DQB1*03:03 & 2 & 13 & $44(2.3-829)$ & 0.01 \\
\hline DQB1*04:01 & 2 & 23 & $0.36(0.12-1.04)$ & 0.05 \\
\hline DQB1 $* 05: 01$ & 3 & 6 & $3(1.3-7)$ & 0.008 \\
\hline
\end{tabular}

Table 3. Comparison of alleles of HLA I and II between Poncet's disease and rheumatoid arthritis.

\begin{tabular}{|c|c|c|c|c|}
\hline \multirow{2}{*}{ HLA I and II } & \multicolumn{2}{|c|}{ Poncet's disease } & \multicolumn{2}{|c|}{ Rheumatoid arthritis } \\
\hline & OR (IC) ${ }^{\circ}$ & $p<0.05$ & OR (IC) ${ }^{\circ}$ & $p<0.05$ \\
\hline$A^{*} 24: 02$ & $4.5(2.7-7.5)$ & 0.0001 & $1.85(0.97-3.5)$ & 0.05 \\
\hline$B^{*} 15: 55$ & $44.5(2.39-829)$ & 0.01 & 0 & 0 \\
\hline$B^{*} 27: 01$ & 44.5 (2.39-829) & 0.01 & $11(0.61-197.8)$ & 0.1038 \\
\hline$B * 35: 01$ & $2.6(1.3-5.3)$ & 0.009 & $0.9(0.49-1.7)$ & 0.8607 \\
\hline$B * 38: 03$ & $70(4.0-1216)$ & 0.0035 & 0 & 0 \\
\hline DQB1*03:01 & $8917(171-4633)$ & 0.0009 & 1.9 (1.17 a 3.28$)$ & 0.01 \\
\hline $\mathrm{DRB}^{*} 14: 14$ & $28(1.4-479)$ & 0.028 & 0 & 0 \\
\hline $\mathrm{DQB1}$ *02:01 & $31(1.5-638)$ & 0.023 & 0 & 0 \\
\hline DQB1*03:03 & $44(2.3-829)$ & 0.01 & 0 & 0 \\
\hline DQB1*05:01 & $3(1.3-7)$ & 0.008 & $2(1.05-3.79)$ & 0.0341 \\
\hline
\end{tabular}

of its association [24]. This polymorphism has functional relevance since the macrophages of patients that posses it have a decreased survival to the experimental mycobacterial infection [25].

The allele $\mathrm{DQB} 1^{*} 0601$ showed a frequency of $16.6 \%$ in patients with RA. This form of the gene has been associated with TB in a meta-analysis of several populations [26]. Furthermore, a subgroup analysis of another study that pooled the effect size of several reports found that the presence of HLA-DQB ${ }^{*} 0601$ rises the risk of the disease 1.68 times in patients with an Asian genetic background [22]. For instance, Mexican mestizo population is composed of Amerindian, African and European genes. In the case of Amerindian genes, these are closely related to the ones of Asian ancestry. On the other side, DQB1*0601 was the allele that showed the strongest association to protection from RA in the study population. According to one hypothesis, RA may have originated from the selection of genes that provided protection against TB [27].
Similarly, those alleles that guard from RA may also increase the susceptibility to TB.

The proportion of patients positive for one of the variants of the DR4 serotype was equal in patients and in controls (43.3\%). This variant has been associated to an increased risk of TB in Mexicans [21]. However, a recent study in a Brazilian population showed an inverse relation between HLA-DRB $1^{*} 04$ and the prevalence of TB [28] which is in accordance to a metaanalysis [24].

For the case of the serotype DR8, $8 \%$ of the patients had the DRB1*0801 allele which have shown protection in a Mexican population [21]. A simulation study supports the hypothesis that the protein product of the HLA-DRB $1 * 0801$ is inefficient at binding antigens from $M . t b$ [29]. The fact that this allele had a higher prevalence in controls as compared to patients with RA supports the hypothesis that there is an interaction between the genes that protect from $\mathrm{RA}$ and that increase the risk to TB. 
A recent meta-analysis identified the HLADRB1*11 allele to be a protective factor to develop TB [24]. In the study population, 6\% of patients with RA and $21 \%$ of controls had a DRB*11 allele, making it a protective factor to develop RA (OR 0.26 [CI: 0.97-0.63], $\mathrm{P}=0.0009)$. This protective association has been described previously [30].

In the research population, we have found several alleles that are disproportionally associated with the presence of RA. These alleles are: are $A^{*} 24: 02, \quad A^{*} 33: 01, \quad B^{*} 08: 01, \quad D Q B 1 * 0403$, DQB1*05:01, DRB1*0302 and DR*B1*1402. Some alterations in the immune regulation associated to RA confer an elevated risk to infections. Our study confirms the associations previously found in other populations [31,32]. For instance, malfunction in the identification and presentation of antigens may leave the host unprotected to fight infections. However, the mechanisms that govern this interaction are poorly characterized. Some of the alleles that we found to be related to the development of RA may be also play a role in the altered response to infections.

One limitation of this study is that it only considered patients with RA. Future studies on the subject should consider other populations that are exposed to biologics to dissect the interaction between the physiopathology of the disease and the treatment. A follow-up study that confirms the diagnosis of TB will test the hypothesis that the risk of developing the disease is higher in patients that have risk alleles and lower in patients with protective variants of the HLA genes. Future studies should also consider other risk factors that have been associated to $\mathrm{TB}$, including diabetes, alcoholism, tobacco consumption, and their interaction to the different genotypes.

\section{Conclusion}

Patients with RA have an increased risk to develop TB and severe complications derived from it. This infectious process is related to the use of biologics. In the era of personalized medicine, a mechanism to identify patients at risk to develop $\mathrm{TB}$ could derive from the findings of genetic studies. In a representative Mexican population, we have identified several alleles of the HLA locus that could advance this technology. In order to confirm its utility, clinical cohort studies need to be conducted.

\section{References}

1. O'Shea JJ, Laurence A, McInnes IB. Back to the future oral targeted therapy for RA and other autoimmune diseases. Nat. Rev. Rheumatol. 9(3), 173-182 (2013).

2. Choy EH, Kavanaugh AF, Jones SA. The problem of choice current biologic agents and future prospects in RA. Nat. Rev Rheumatol. 9(3), 154-163 (2013).

3. Winthrop KL, Chiller T. Preventing and treating biologic-associated opportunistic infections. Nat. Rev. Rheumatol. 5(7), 405-410 (2009).

4. Dolin PJ, Raviglione MC, Kochi A. Global tuberculosis incidence and mortality during 1990-2000. Bull World Health Organ 72, 213 220 (1994).

5. Winthrop KL, Iseman M. Bedfellows: mycobacteria and rheumatoid arthritis in the era of biologic therapy. Nat. Rev. Rheumatol. 9, 524-531 (2013).

6. Frieden TR, Sterling TR, Munsiff SS et al. Tuberculosis. Lancet. 362(9387), 887-899 (2003).

7. Wolfe F, Michaud K, Anderson J et al. Tuberculosis infection in patients with rheumatoid arthritis and the effect of infliximab therapy. Arthritis. Rheum. 50(2), 372-379 (2004).

8. Winthrop KL, Park S-H, Gul A et al. Tuberculosis and other opportunistic infections in tofacitinibtreated patients with rheumatoid arthritis. Ann. Rheum. Dis. 75(6), 1133-1138 (2016).

9. Abel L, El-Baghdadi J, Bousfiha AA et al. Human genetics of tuberculosis: a long and winding road. Philos. Trans. R. Soc. Lond. B. Biol. Sci. 19, 1645 (2014).

10. Philips JA, Ernst JD. Tuberculosis Pathogenesis and Immunity. Annu. Rev. Pathol. Mech. Dis. 7(1), 353-384 (2012).

11. Lykouras D, Sampsonas F, Kaparianos A et al. Human genes in TB infection: their role in immune response. Monaldi. Arch. Chest. Dis. 69(1), 24-31 (2008).

12. Dubaniewicz A, Lewko B, Moszkowska G et al. Molecular subtypes of the HLA-DR antigens in pulmonary tuberculosis. Int. J. Infect. Dis. Elsevier. 4(3), 129-133 (2000).

13. Alisherov AS, Kitaev MI, Tarasenko OM et al. HLA genes in patients with pulmonary tuberculosis in the Kirghiz population. Probl. Tuberk. 5, 41-42 (1997).

14. Singh SP, Mehra NK, Dingley HB et al. Human leukocyte antigen (HLA)-linked control of susceptibility to pulmonary tuberculosis and association with HLA-DR types. J. Infect. Dis. 
148(4), 676-681 (1983).

15. Goldfeld AE, Delgado JC, Thim $S$ et al. Association of an HLA-DQ allele with clinical tuberculosis. JAMA. 21, 226-228 (1998).

16. Lugo-Zamudio GE, Yamamoto-Furusho JK, Delgado-Ochoa D et al. Human leukocyte antigen typing in tuberculous rheumatism: Poncet's disease. Int. J. Tuberc. Lung. Dis. 14, 916-920 (2010).

17. Yarwood A, Huizinga TWJ, Worthington J. The genetics of rheumatoid arthritis: Risk and protection in different stages of the evolution of RA. Rheumatol (United Kingdom). 55(2), 199209 (2016).

18. Vargas-Alarcón G, Granados J, Rodríguez-Pérez JM et al. Distribution of HLA class II alleles and haplotypes in Mexican Mestizo population: comparison with other populations. Immunol. Invest. 39(3), 268-283 (2010).

19. Gourraud P-A, Hollenbach JA, Barnetche T et al. Standard methods for the management of immunogenetic data. Methods. Mol. Biol. 882, 197-213 (2012).

20. Marsh SGE, Albert ED, Bodmer WF et al. Nomenclature for factors of the HLA system, 2010. Tissue. Antigens. 75(2), 291-455 (2010).

21. Terán-Escandón D, Terán-Ortiz L, CamarenaOlvera A et al. Human leukocyte antigenassociated susceptibility to pulmonary tuberculosis: Molecular analysis of class II alleles by DNA amplification and oligonucleotide hybridization in Mexican patients. Chest. 115(2), 428-433 (1999).

22. L Li C, Zhou Y, Xiang X et al. The Relationship of HLA-DQ Alleles with Tuberculosis Risk: A Meta-analysis. Lung [Internet]. 193(4), 521-530 (2015).

23. Ravikumar M, Dheenadhayalan V, Rajaram K et al. Associations of HLA-DRB1, DQB1 and DPB1 alleles with pulmonary tuberculosis in south India. Tuber. Lung. Dis. 79(5), 309-317 (1999).
24. Tong X, Chen L, Liu S et al. Polymorphisms in HLA-DRB1 Gene and the Risk of Tuberculosis: A Meta-analysis of 31 Studies. Lung. 193(2), 309-318 (2015).

25. Singh AK, Abhimanyu, Yadav $A B$ et al. HLADRB1*1501 and VDR polymorphisms and survival of Mycobacterium tuberculosis in human macrophages exposed to inhalable microparticles. Pharmacogenomics. 14(5), 531-540 (2013).

26. Oliveira-Cortez A, Melo AC, Chaves VE et al. Do HLA class II genes protect against pulmonary tuberculosis? A systematic review and metaanalysis. Eur. J. Clin. Microbiol. Infect. Dis. 35(10), 1567-1580 (2016).

27. Mobley JL. Is rheumatoid arthritis a consequence of natural selection for enhanced tuberculosis resistance? Med. Hypotheses. 62(5), 839-843 (2004).

28. De Lima DS, Ogusku MM, Dos Santos MP et al. Alleles of HLA-DRB $1 * 04$ associated with pulmonary tuberculosis in Amazon Brazilian population. PLoS One. 11(2), 2-e0147543 (2016).

29. Contini S, Pallante M, Vejbaesya S et al. A model of phenotypic susceptibility to tuberculosis: deficient in silico selection of Mycobacterium tuberculosis epitopes by HLA alleles. Sarcoidosis. Vasc. Diffuse. Lung. Dis. 25, 21-28 (2008).

30. Mourad J, Monem F. HLA-DRB1 allele association with rheumatoid arthritis susceptibility and severity in Syria. Rev. Bras. Reumatol. 53(1), 47-56 (2013).

31. Raychaudhuri S, Sandor C, Stahl EA et al. Five amino acids in three HLA proteins explain most of the association between MHC and seropositive rheumatoid arthritis. Nat. Genet. 44(3), 291-296 (2012).

32. Han B, Diogo D, Eyre $S$ et al. Fine mapping seronegative and seropositive rheumatoid arthritis to shared and distinct HLA alleles by adjusting for the effects of heterogeneity. Am. J. Hum. Genet. 94, 522-532 (2014). 